\title{
Automated Assume-Guarantee Reasoning by Abstraction Refinement
}

\author{
Mihaela Gheorghiu Bobaru ${ }^{1,2}$, Corina S. Păsăreanu ${ }^{1}$, and Dimitra Giannakopoulou ${ }^{1}$ \\ ${ }^{1}$ PSGS and RIACS, NASA Ames Research Center, \\ Moffett Field, CA 94035, USA \\ ${ }^{2}$ Department of Computer Science, University of Toronto, \\ Toronto, ON M5S 3G4, Canada \\ mg@cs.toronto.edu, \\ \{corina.s.pasareanu, dimitra.giannakopoulou\}@nasa.gov
}

\begin{abstract}
Current automated approaches for compositional model checking in the assume-guarantee style are based on learning of assumptions as deterministic automata. We propose an alternative approach based on abstraction refinement. Our new method computes the assumptions for the assume-guarantee rules as conservative and not necessarily deterministic abstractions of some of the components, and refines those abstractions using counterexamples obtained from model checking them together with the other components. Our approach also exploits the alphabets of the interfaces between components and performs iterative refinement of those alphabets as well as of the abstractions. We show experimentally that our preliminary implementation of the proposed alternative achieves similar or better performance than a previous learning-based implementation.
\end{abstract}

\section{Introduction}

Despite impressive recent progress in the application of model checking to the verification of realistic systems, the essential challenge in model checking remains the wellknown state-space explosion problem [8]. Compositional techniques attempt to tame this problem by applying verification to individual components and merging the results without analyzing the whole system. In checking components individually, it is often necessary to incorporate some knowledge of the context in which each component is expected to operate correctly. Assume-guarantee reasoning [13|15] addresses this issue by using assumptions that capture the expectations that a component makes about its environment. Assumptions have traditionally been developed manually, which has limited the practical impact of assume-guarantee reasoning.

In recent work, automation has been achieved through learning-based techniques [10]. The $\mathrm{L}^{*}$ learning algorithm [2] is used to generate the assumptions needed for the assumeguarantee rules. The simplest such rule checks if a system composed of components $M_{1}$ and $M_{2}$ satisfies a property $P$ by checking that $M_{1}$ under assumption $A$ satisfies $P$ (Premise 1 ) and discharging $A$ on the environment $M_{2}$ (Premise 2). For safety properties, Premise 2 amounts to checking that $A$ is a conservative abstraction of $M_{2}$, i.e., an abstraction that preserves all of $M_{2}$ 's execution paths. This rule is also represented as follows, where the notation is described in more detail in Section 2.

A. Gupta and S. Malik (Eds.): CAV 2008, LNCS 5123, pp. 135-148, 2008.

(C) Springer-Verlag Berlin Heidelberg 2008 


$$
\begin{aligned}
& \text { (Premise 1) }\langle A\rangle M_{1}\langle P\rangle \\
& \frac{\text { (Premise 2) }\langle\text { true }\rangle M_{2}\langle A\rangle}{\langle\text { true }\rangle M_{1} \| M_{2}\langle P\rangle}
\end{aligned}
$$

Learning-based assume-guarantee verification is an iterative process, during which $\mathrm{L}^{*}$ makes conjectures in the form of automata that represent intermediate assumptions. Each conjectured assumption $A$ is used to check the two premises of Rule 1 The process ends if $A$ passes both premises of the rule, in which case the property holds in the system, or if it uncovers a real violation. Otherwise, a counterexample is returned and $\mathrm{L}^{*}$ modifies the conjecture. Similar approaches are proposed in [14]17]; the work in [12] uses sampling rather than $\mathrm{L}^{*}$ to learn the assumptions in a similar way.

In this paper we propose an alternative approach, AGAR (Assume-Guarantee Abstraction Refinement), that automates assume-guarantee reasoning by iteratively computing assumptions as conservative abstractions of the interface behavior of $M_{2}$, i.e., the behavior that concerns the interaction with $M_{1}$. In each iteration, the computed assumption $A$ satisfies Premise 2 of the Rule1 by construction and it is only checked for Premise 1. If the check is successful, we conclude that $M_{1} \| M_{2}$ satisfies the property; if the check fails, we get a counterexample trace that we analyze to see if it corresponds to a real error in $M_{1} \| M_{2}$ or it is spurious due to the over-approximation in the abstraction. If it is spurious, we used it to refine $A$ and then repeat the entire process. Unlike learning-based assumption generation, AGAR does not constrain assumptions to be deterministic. Therefore the assumptions constructed with AGAR can be (potentially) exponentially smaller than those obtained with learning, resulting in smaller verification problems.

To reduce the assumption sizes even further, we also combine the abstraction refinement with an orthogonal technique, interface alphabet refinement, which extends AGAR so that it starts the construction of $A$ with a small subset of the interface alphabet and adds actions to the alphabet as necessary until the required property is shown to hold or to be violated in the system. Actions to be added are discovered also by counterexample analysis. We introduced alphabet refinement in [11] for learning-based assumeguarantee reasoning; we adapt it here for AGAR 1 . We have implemented AGAR with alphabet refinement in the explicit state model checker LTSA [14] and performed a series of experiments that demonstrate that it can achieve better performance than $\mathrm{L}^{*}$ for Rule 1 above.

Related work. AGAR is a variant of the well-known CEGAR (Counter ExampleGuided Abstraction Refinement) [7] with the notable differences that the computed abstractions keep information only about the interface behavior of $M_{2}$ that concerns the interaction with $M_{1}$ while it abstracts away its internal behavior, and that the counterexamples used for the refinement of $M_{2}$ 's abstractions are obtained in an assumeguarantee style by model checking the other component, $M_{1}$.

CEGAR has been used before in compositional reasoning in [5]). In that work, a conservative abstraction of every component is constructed and then all the resulting abstractions are composed and checked. If the check passes, the verification concludes successfully, otherwise the resulting abstract counterexample is analyzed on every

\footnotetext{
${ }^{1}$ Note that [6] introduced a related alphabet minimization technique for $\mathrm{L}^{*}$ as well.
} 


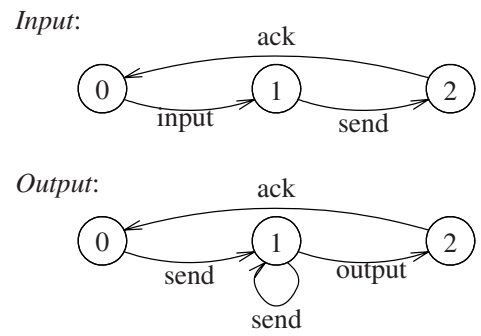

a)

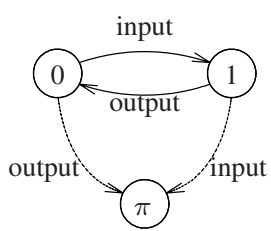

b)

Fig. 1. (a) Example LTSs; (b) Order property

abstraction that is refined if needed. The work does not use assume-guarantee reasoning, it does not address the reduction of the interface alphabets and it has not been compared with learning-based techniques.

A comparison of learning and CEGAR-based techniques has been performed in [3] but for a different problem: the "interface synthesis" for a single component whose environment is unknown. In our context, this would mean generating an assumption that passes Premise 1, in the absence of a second component against which to check Premise 2. The interface being synthesized by the CEGAR-based algorithm in [3] is built as an abstraction of $M_{1}$. The work does not apply reduction to interface alphabets, nor does it address the verification of the generated interfaces against other components, i.e., completing the assume-guarantee reasoning.

\section{Preliminaries}

Labeled Transition Systems (LTSs). We model components as finite-state labeled transition systems (LTSs), as considered by LTSA. Let $\mathbf{U}$ be the universal set of observable actions and let $\tau$ denote a special action that is unobservable.

An LTS $M$ is a tuple $\left\langle Q, \Sigma, \delta, q_{0}\right\rangle$, where: $Q$ is a finite non-empty set of states; $\Sigma \subseteq \mathbf{U}$ is the alphabet of $M ; \delta \subseteq Q \times(\Sigma \cup\{\tau\}) \times Q$ is a transition relation, and $q_{0}$ is the initial state. We write $\left(q, a, q^{\prime}\right) \in \delta$ as $q \stackrel{a}{\rightarrow} q^{\prime}$. An LTS $M$ is non-deterministic if it contains $\tau$-transitions or if $\exists\left(q, a, q^{\prime}\right),\left(q, a, q^{\prime \prime}\right) \in \delta$ such that $q^{\prime} \neq q^{\prime \prime}$. Otherwise, $M$ is deterministic. $\pi$ denotes an error state with no outgoing transitions, and $\Pi$ denotes the LTS $\langle\{\pi\}, \mathbf{U}, \emptyset, \pi\rangle$. Let $M=\left\langle Q, \Sigma, \delta, q_{0}\right\rangle$ and $M^{\prime}=\left\langle Q^{\prime}, \Sigma^{\prime}, \delta^{\prime}, q_{0}^{\prime}\right\rangle ; M$ transits into $M^{\prime}$ with action $a$, denoted $M \stackrel{a}{\rightarrow} M^{\prime}$, if $\left(q_{0}, a, q_{0}^{\prime}\right) \in \delta$ and either $Q=Q^{\prime}, \Sigma=\Sigma^{\prime}$, and $\delta=\delta^{\prime}$ for $q_{0}^{\prime} \neq \pi$, or, in the special case where $q_{0}^{\prime}=\pi, M^{\prime}=\Pi$.

Parallel Composition. Parallel composition " $\| "$ is a commutative and associative operator such that: given LTSs $M_{1}=\left\langle Q_{1}, \Sigma_{1}, \delta^{1}, q_{0}^{1}\right\rangle$ and $M_{2}=\left\langle Q_{2}, \Sigma_{2}, \delta^{2}, q_{0}^{2}\right\rangle$, $M_{1} \| M_{2}$ is $\Pi$ if either one of $M_{1}, M_{2}$ is $\Pi$. Otherwise, $M_{1} \| M_{2}$ is an LTS $M=\left\langle Q, \Sigma, \delta, q_{0}\right\rangle$ where $Q=Q_{1} \times Q_{2}, q_{0}=\left(q_{0}^{1}, q_{0}^{2}\right), \Sigma=\Sigma_{1} \cup \Sigma_{2}$, and $\delta$ is defined as follows (the symmetric version also applies): $M_{1}\left\|M_{2} \stackrel{a}{\rightarrow} M_{1}^{\prime}\right\| M_{2}$ if $M_{1} \stackrel{a}{\rightarrow} M_{1}^{\prime}, a \notin \Sigma_{2}$, and $M_{1}\left\|M_{2} \stackrel{a}{\rightarrow} M_{1}^{\prime}\right\| M_{2}^{\prime}$ if $M_{1} \stackrel{a}{\rightarrow} M_{1}^{\prime}, M_{2} \stackrel{a}{\rightarrow} M_{2}^{\prime}, a \neq \tau$. 
As an example [10], consider a simple communication channel that consists of two components whose LTSs are shown in Fig.11(a).

Paths and traces. A path in an LTSs $M=\left\langle Q, \Sigma, \delta, q_{0}\right\rangle$ is a sequence $p$ of alternating states and (observable or unobservable actions) of $M, p=q_{i_{0}}, a_{0}, q_{i_{1}}, a_{1}, \ldots, a_{n-1}, q_{i_{n}}$ such that for every $k \in\{0, \ldots, n-1\}$ we have $\left(q_{i_{k}}, a_{k}, q_{i_{k+1}}\right) \in \delta$.

The trace of path $p$, denoted $\sigma(p)$ is the sequence $b_{0}, b_{1}, \ldots, b_{l}$ of actions along $p$, obtained by removing all $\tau$ from $a_{0}, \ldots, a_{n-1}$. A state $q$ reaches a state $q^{\prime}$ in $M$ with a sequence of actions $t$, denoted $q \stackrel{t}{\Rightarrow} q^{\prime}$, if there exists a path $p$ from $q$ to $q^{\prime}$ in $M$ whose trace is $t$, i.e., $\sigma(p)=t$. A trace of $M$ is the trace of a path in $M$ starting from $q_{0}$. The set of all traces of $M$ forms the language of $M$, denoted $\mathcal{L}(M)$. For any trace $t=a_{0}, a_{1}, \ldots, a_{n-1}$, a trace LTS can be constructed whose only transitions are $q_{0} \stackrel{a_{0}}{\longrightarrow} q_{1} \stackrel{a_{1}}{\longrightarrow} q_{2} \ldots \stackrel{a_{n-1}}{\longrightarrow} q_{n}$. We sometimes abuse the notation and denote by $t$ both a trace and its trace LTS. The meaning should be clear from the context. For $\Sigma^{\prime} \subseteq \Sigma$, $t \downarrow_{\Sigma^{\prime}}$ is the trace obtained by removing from $t$ all actions $a \notin \Sigma$. Similarly, $M \downarrow_{\Sigma^{\prime}}$ is an LTS over $\Sigma$ obtained from $M$ by renaming to $\tau$ all the action labels not in $\Sigma$. Let $t_{1}, t_{2}$ be two traces. Let $\Sigma_{1}, \Sigma_{2}$ be the sets of actions occurring in $t_{1}, t_{2}$, respectively. By the symmetric difference of $t_{1}$ and $t_{2}$ we mean the symmetric difference of sets $\Sigma_{1}$ and $\Sigma_{2}$.

Safety properties. A safety LTS is a deterministic LTS not containing $\pi$. A safety property $P$ is a safety LTS whose language $\mathcal{L}(P)$ defines the acceptable behaviors over $\Sigma_{P}$.

An LTS $M=\left\langle Q, \Sigma, \delta, q_{0}\right\rangle$ satisfies $P=\left\langle Q_{P}, \Sigma_{P}, \delta_{P}, q_{0}^{P}\right\rangle$, denoted $M \models P$, iff $\forall t \in \mathcal{L}(M) \cdot t \downarrow_{\Sigma_{P}} \in \mathcal{L}(P)$. For checking a property $P$, its safety LTS is completed by adding error state $\pi$ and transitions on all the missing outgoing actions from all states into $\pi$ so that the resulting transition relation is (left-)total (when seen as in $(Q \times$ $(\Sigma \cup\{\tau\})) \times Q$ ) and deterministic; the resulting LTS is denoted by $P_{\text {err }}$. LTSA checks $M \models P$ by computing $M \| P_{\text {err }}$ and checking if $\pi$ is reachable in the resulting LTS.

For example, the Order property in Fig. 1(b) states that inputs and outputs come in matched pairs, with the input always preceding the output. The dashed arrows represent transitions to the error state that were added to obtain rder $_{\text {err }}$.

Assume-guarantee triples. An assume-guarantee triple $\langle A\rangle M\langle P\rangle$ is true if whenever component $M$ is part of a system satisfying assumption $A$, the system must also guarantee property $P$. In LTSA, this reduces to checking whether $A \| M \models P$.

Learning assumptions with $\mathbf{L}^{*}$. Previous work [10] uses the $\mathrm{L}^{*}$ algorithm [2] to iteratively learn the assumption $A$ for Rule 1 as a deterministic finite state automaton. $\mathrm{L}^{*}$ needs to interact with a teacher that answers queries and validates conjectures. For membership queries on string $s$, the teacher uses LTSA to check $\langle s\rangle M_{1}\langle P\rangle$; if true, then $s \in \mathcal{L}(A)$ and the Teacher returns "true". Otherwise, the answer to the query is "false". The conjectures returned by $\mathrm{L}^{*}$ are intermediate assumptions; the teacher implements two oracles to validate these conjectures: Oracle 1 guides L* towards a conjecture that makes $\langle A\rangle M_{1}\langle P\rangle$ true and then Oracle 2 is invoked to discharge $A$ on $M_{2}$. If this is also true, then the assume guarantee rule ensures that $P$ holds on $M_{1} \| M_{2}$; the teacher returns "true" and the computed assumption $A$. If model checking returns "false", the returned counterexample is analyzed to determine if $P$ is indeed violated in $M_{1} \| M_{2}$ or if $A$ is imprecise due to learning, in which case $A$ is modified 
and the process repeats. If $A$ has $n$ states, $\mathrm{L}^{*}$ makes at most $n-1$ incorrect conjectures. The number of membership queries made by $\mathrm{L}^{*}$ is $O\left(k n^{2}+n \log m\right)$, where $k$ is the size of $A$ 's alphabet and $m$ is the length of the longest counterexample returned when a conjecture is made.

Interface alphabet. When reasoning in an assume-guarantee style, there is a natural notion of the complete interface between $M_{1}$ and $M_{2}$, when property $P$ is checked. Let $M_{1}=\left\langle Q_{1}, \Sigma_{1}, \delta^{1}, q_{0}^{1}\right\rangle$ and $M_{2}=\left\langle Q_{2}, \Sigma_{2}, \delta^{2}, q_{0}^{2}\right\rangle$ be LTSs modeling two components and let $P=\left\langle Q_{P}, \Sigma_{P}, \delta^{P}, q_{0}^{P}\right\rangle$ be a safety property. The interface alphabet $\Sigma_{I}$ is defined as $\Sigma_{I}=\left(\Sigma_{1} \cup \Sigma_{P}\right) \cap \Sigma_{2}$.

\section{Motivating Example}

We motivate our approach using the input-output example from Section 2 . We show that even on this simple example AGAR leads to smaller assumptions in fewer iterations than the learning approach, and therefore it potentially leads faster to smaller verification problems.

Let $M_{1}=$ Input, $M_{2}=$ Output, and $P=$ Order. As mentioned, we aim to automatically compute an assumption according to Rule 1. Instead of "guessing" an assumption and then checking both premises of the rule, as in the learning approaches, we build an abstraction that satisfies Premise 2 by construction. Therefore, all that needs to be checked is Premise 1.

The initial abstraction $A$ of Output is illustrated in Figure 2 (a). Its alphabet consists of the interface between Input and the Order property on one side, and Output on the other, i.e., the alphabet of $A$ is $\Sigma_{I}=\left\{\left(\Sigma_{\text {Input }} \cup \Sigma_{\text {Order }}\right) \cap \Sigma_{\text {Output }}\right.$. The LTS $A$ is constructed simply by mapping all concrete states in Output to the same abstract state 0 which has a self-loop on every action in $\Sigma_{I}$ and no other transitions. By construction, $A$ is an overapproximation of $M_{2}$, i.e., $\mathcal{L}\left(M_{2} \downarrow_{\Sigma_{I}}\right) \subseteq \mathcal{L}(A)$, and therefore Premise 2 $\langle$ true $\rangle M_{2}\langle A\rangle$ holds. Checking Premise 1 of the assume-guarantee rule using $A$ as the assumption fails, with abstract counterexample: 0 , output, 0 . We simulate this counterexample on $M_{2}$ and find that it is spurious (i.e., it does not correspond to a trace in $M_{2}$ ), therefore $A$ needs to be refined so that the refined abstraction no longer contains this trace. We split abstract state 0 into two new abstract states: abstract state 0 , representing concrete states 0 and 2 that do not have an outgoing output action, and abstract state 1 , representing concrete state 1 that has an outgoing output action, and adjust the transitions accordingly. The refined abstraction $A^{\prime}$, shown in Figure 2 a), is checked again for Premise 1 and this time it passes, therefore AGAR terminates and reports that the property holds.

The sequence of assumptions learned with $\mathrm{L}^{*}$ is shown in Figure2(b). The assumption computed by AGAR thus has two states fewer than that obtained from learning and is computed in two fewer iterations.

\section{Assume-Guarantee Abstraction Refinement (AGAR)}

The abstraction refinement presented here is an adaptation of the CEGAR framework of [7], with the following notable differences: 1) abstraction refinement is performed 
A:

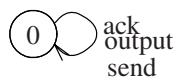

$A^{\prime}$ :

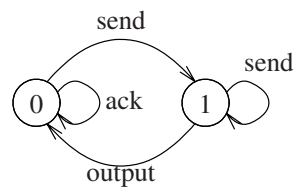

(a)
$A_{0}:$
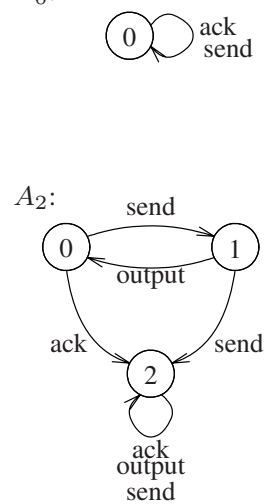
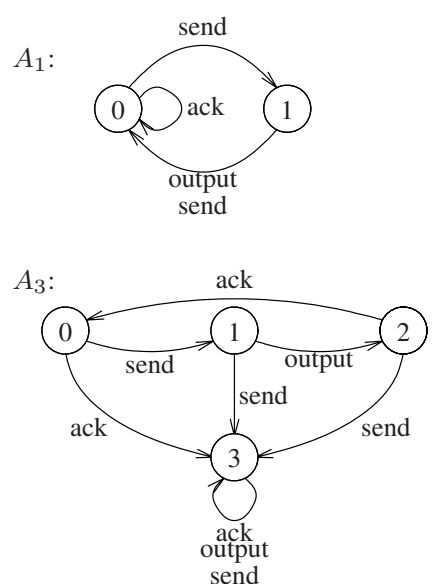

(b)

Fig. 2. Assumptions computed (a) with our algorithm and (b) with $\mathrm{L}^{*}$

in the context of LTSs; abstract transitions for LTSs are computed using closure with respect to actions that are not in their interface alphabet, and 2) counterexample analysis is performed in an assume-guarantee style: a counterexample obtained from model checking one component is used to refine abstractions of a different component.

In this section, we start by describing, independently of the assume-guarantee rule, abstraction refinement as applied to LTSs. We then describe how we use this abstraction refinement in an iterative algorithm (AGAR) that computes assumptions for Rule 1 Later on, we combine AGAR with an orthogonal algorithm that performs iterative refinement of the interface alphabet between the analyzed components.

\subsection{Abstraction Refinement for LTSs}

Abstraction. Let $C=\left\langle Q_{C}, \Sigma_{C}, \delta^{C}, q_{0}^{C}\right\rangle$ be an LTS that we refer to as concrete. Let alphabet $\Sigma_{A}$ be such that $\Sigma_{A} \subseteq \Sigma_{C}$. An abstraction $A$ of $C$ is an LTS $\left\langle Q_{A}, \Sigma_{A}, \delta^{A}, q_{0}^{A}\right\rangle$ such that there exists a surjection $\alpha: Q_{C} \rightarrow Q_{A}$, called the abstraction function, that maps each concrete state $q^{C} \in Q_{C}$ to an abstract state $q^{A} \in Q_{A} ; q_{0}^{A}$ must be such that $\alpha\left(q_{0}^{C}\right)=q_{0}^{A}$. The concretization function $\gamma: Q_{A} \rightarrow 2^{Q_{C}}$ is defined for any $q^{A} \in Q_{A}$ as $\gamma\left(q^{A}\right)=\left\{q^{C} \in Q_{C} \mid \alpha\left(q^{C}\right)=q^{A}\right\}$. Note that $\gamma$ induces a partition on $Q_{C}$, namely $\left\{\gamma\left(q^{A}\right) \mid q^{A} \in Q_{A}\right\}$.

To define the abstract transition relation $\delta^{A}$, we first introduce the notion of reachability with respect to a subset alphabet. For $q^{C} \in C, a \in \Sigma_{C}$, we define the set Reachable $_{C}\left(q^{C}, a, \Sigma_{A}\right)$ of concrete states $q_{i}^{C}$ reachable from $q^{C}$ on action $a$, under the transitive closure of $\delta^{C}$ over actions in $\left(\Sigma_{C} \backslash \Sigma_{A}\right) \cup\{\tau\}$ :

Reachable $_{C}\left(q^{C}, a, \Sigma_{A}\right)=\left\{q_{i}^{C} \in C \mid \exists t, t^{\prime} \in\left(\left(\Sigma_{C} \backslash \Sigma_{A}\right) \cup\{\tau\}\right)^{*} \cdot q^{C} \stackrel{t}{\Rightarrow} q_{i}^{C}\right.$ or $\left.q^{C} \stackrel{t, b, t^{\prime}}{\Rightarrow} q_{i}^{C}\right\}$. 


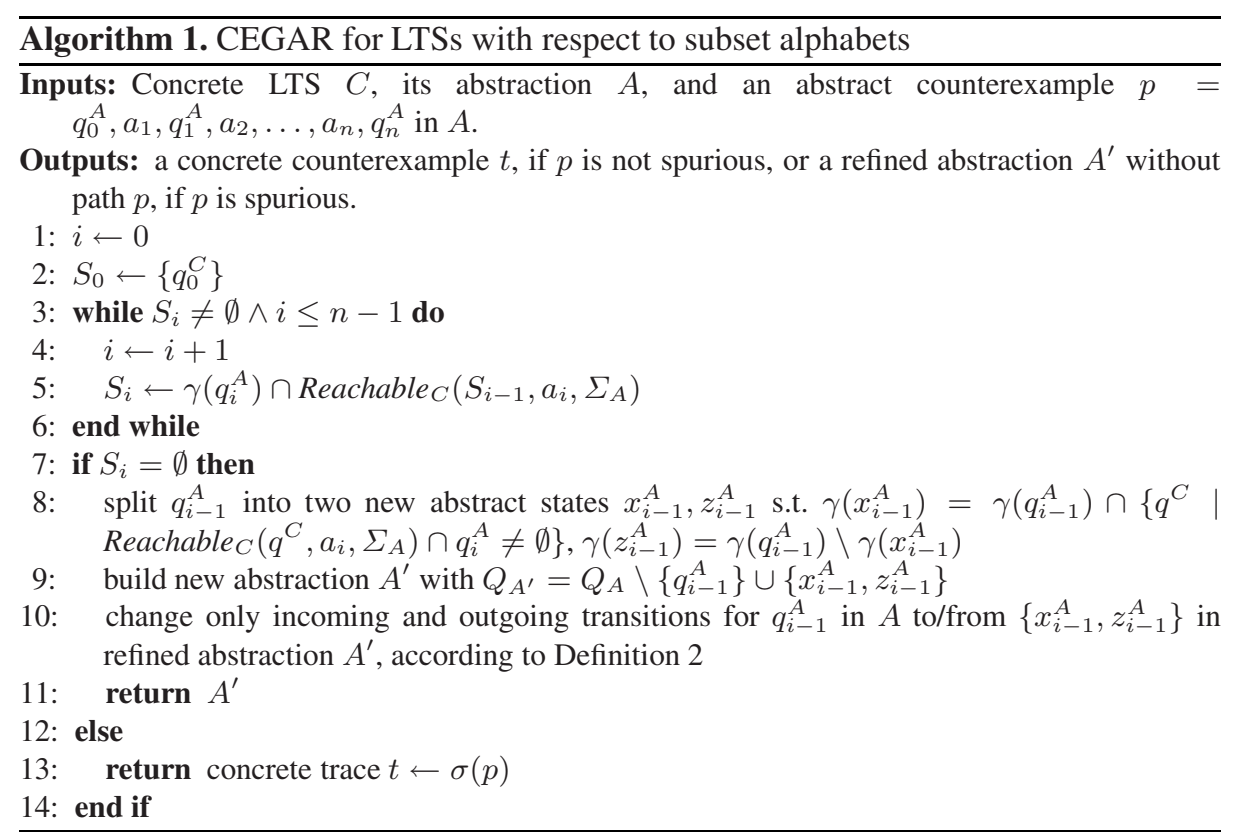

We define the abstraction to be existential, but using Reachable $_{C}$ instead of the usual transition relation of $C$ [7]: $\exists\left(q_{i}^{A}, a, q_{j}^{A}\right) \in \delta^{A}$ iff

$$
\exists q_{i}^{C}, q_{j}^{C} \in C \cdot \alpha\left(q_{i}^{C}\right)=q_{i}^{A}, \alpha\left(q_{j}^{C}\right)=q_{j}^{A}, \text { and } q_{j}^{C} \in \text { Reachable }_{C}\left(q_{i}^{C}, a, \Sigma_{A}\right)
$$

From the above definition and that of weak simulation [16], it follows that the abstraction defines a weak simulation relation between $C \downarrow_{\Sigma_{A}}$ and $A$. It is known that weak simulation implies trace inclusion [16]. We therefore have the following:

Proposition 1. Given concrete LTS $C$ and and its abstraction $A$ defined as above, $\mathcal{L}\left(C \downarrow_{\Sigma_{A}}\right) \subseteq \mathcal{L}(A)$, and consequently $\langle$ true $\rangle C\langle A\rangle$ hold.

The CEGAR algorithm for LTSs is defined by Algorithm 1 It takes as inputs a concrete system $C$, an abstraction $A$ (as defined above), and an abstract counterexample path $p$ (in $A$ ). The algorithm analyzes the counterexample (lines 1-6) to see if it is real, in which case it is returned (line 13) or spurious, in which case it is used to refine the abstraction (lines 7-11). The refined abstraction $A^{\prime}$ is such that it no longer contains $p$. We discuss Algorithm 1 in more detail below.

Analysis of abstract counterexamples. Suppose we have obtained an abstract counterexample in the form of a path $p=q_{0}^{A}, a_{1}, q_{1}^{A}, a_{2}, \ldots, a_{n}, q_{n}^{A}$ in the abstraction $A$ of $C$. We want to determine if it corresponds to a concrete path in $C$. For this we need to "play" (i.e. symbolically simulate) $p$ in $C$ from the initial state $q_{0}^{C}$. We do so considering that $\Sigma_{A} \subseteq \Sigma_{C}$ and thus we use Reachable $_{C}$ again.

We first extend Reachable $_{C}$ to sets: for $S \subseteq Q_{C}$, Reachable $_{C}\left(S, a, \Sigma_{A}\right)=\left\{q_{j}^{C} \in\right.$ $C \mid \exists q_{i}^{C} \in S . q_{j}^{C} \in$ Reachable $\left.\left(q_{i}^{C}, a, \Sigma_{A}\right)\right\}$. We play the abstract counterexample $p$ 
following [7]. We start at step 0 with the set $S_{0}=\left\{q_{0}^{C}\right\}$ of concrete states, and the first transition $q_{0}^{A} \stackrel{a_{1}}{\rightarrow} q_{1}^{A}$ from $p$. Note that $S_{0}=\left\{q_{0}^{C}\right\} \cap \gamma\left(q_{0}^{A}\right)$. At each step $i \in\{1, \ldots, n\}$, we compute the set $S_{i}=\gamma\left(q_{i}^{A}\right) \cap$ Reachable $_{C}\left(S_{i-1}, a_{i}, \Sigma_{A}\right)$. If, for some $i \leq n, S_{i}$ is empty, the abstract counterexample is spurious and we need to refine the abstraction to eliminate it. Otherwise, the counterexample corresponds to a concrete path.

Abstraction refinement. The abstraction refinement is performed in lines 8-10 of Algorithm $1 p$ is spurious because abstract state $q_{i-1}^{A}$ does not distinguish between two disjoint, non-empty sets of concrete states [7]: (i) those that reach, with action $a_{i}$, states in the concretization of $q_{i}^{A}$ (these are the states defined as $\gamma\left(x_{i-1}^{A}\right)$ in line 8) and (ii) those reached so far from $q_{0}^{C}$ with the prefix $a_{1}, a_{2}, \ldots, a_{i-1}$, i.e., the states in $S_{i-1}$.

To eliminate the spurious abstract path, we need to refine $A$ by splitting its state $q_{i-1}^{A}$ into (at least) two new abstract states that separate the (concrete) states of types (i) and (ii) (line 9). We split $q_{i-1}^{A}$ into $x_{i-1}^{A}$ where $\gamma\left(x_{i-1}^{A}\right)$ contains the set of states in (i) and $z_{i-1}^{A}$ where $\gamma\left(z_{i-1}^{A}\right)$ contains the set of states in (ii) and any remaining states in $\gamma\left(q_{i-1}^{A}\right)$. Note that this results in a finer partition of the concrete states. After the splitting, we update the abstract transitions in line 10. The refined abstraction $A^{\prime}$ has the same transitions as $A$ except for those incoming or outgoing for the split state $q_{i-1}^{A}$ : they are readjusted to point to or from the states $x_{i-1}^{A}, z_{i-1}^{A}$ according to condition 2 We therefore can conclude that:

Lemma 1. If a counterexample $p$ input to Algorithm $\square$ is spurious, the returned abstraction $A^{\prime}$ results in a strictly finer partition than $A$ and does not contain $p$.

\subsection{The AGAR Algorithm}

The pseudocode that combines Algorithm 1 with Rule 1 is given in Algorithm 2 Recall that $\Sigma_{I}$ denotes the alphabet $\left(\Sigma_{M_{1}} \cup \Sigma_{P}\right) \cap \Sigma_{M_{2}}$ of the interface between $M_{1}$ and $M_{2}$, with respect to $P$. The algorithm checks that $M_{1} \| M_{2}$ satisfies $P$ using Rule 1 It builds abstractions $A$ of $M_{2}$ in an iterative fashion (while loop at lines 2-15); these abstractions are used to check Premise 1 of the assume guarantee rule using model checking (lines 3-5). If the check is successful, then, according to the rule (and since $A$ satisfies Premise 2 by construction), $P$ indeed holds in $M_{1} \| M_{2}$ and the algorithm returns "true". Otherwise, a counterexample $p$ is obtained from model checking Premise 1 (line 7) and Algorithm 1 is invoked to check if $p$ corresponds to a real path in $M_{2}$ (in which case it means $p$ is a real error in $M_{1} \| M_{2}$ and this is reported to the user in line 11). If $p$ is spurious, Algorithm 1 returns a refined abstraction $A^{\prime}$ for which we repeat the whole process starting from checking Premise 1.

Obtaining an abstract counterexample. As mentioned, we use counterexamples from failed checks of Premise 1 (that involves checking component $M_{1}$ ) to refine abstractions of $M_{2}$. Obtaining an abstract counterexample involves several steps (lines 7-9). First, a counterexample from line 4 is a path $o=q_{0}, b_{1}, q_{1}, b_{2}, \ldots, b_{l}, q_{l}$ in $A\left\|M_{1}\right\| P_{\text {err }}$. Thus, for every $i \in\{0, l\}, q_{i}$ is a triple of states $\left(q_{i}^{A}, q_{i}^{1}, p_{i}\right)$ from $A \times M_{1} \times P_{e r r}$. We first project every triple on $A$ to obtain the sequence $o^{\prime}=q_{0}^{A}, b_{1}, q_{1}^{A}, b_{2}, q_{2}^{A}, \ldots, b_{l} q_{l}^{A}$; $o^{\prime}$ is not yet a path in $A$ as it may contain actions from $M_{1}$ and $P_{e r r}$ that are not observable to $A$; those actions have to be between the same consecutive abstract states in the 


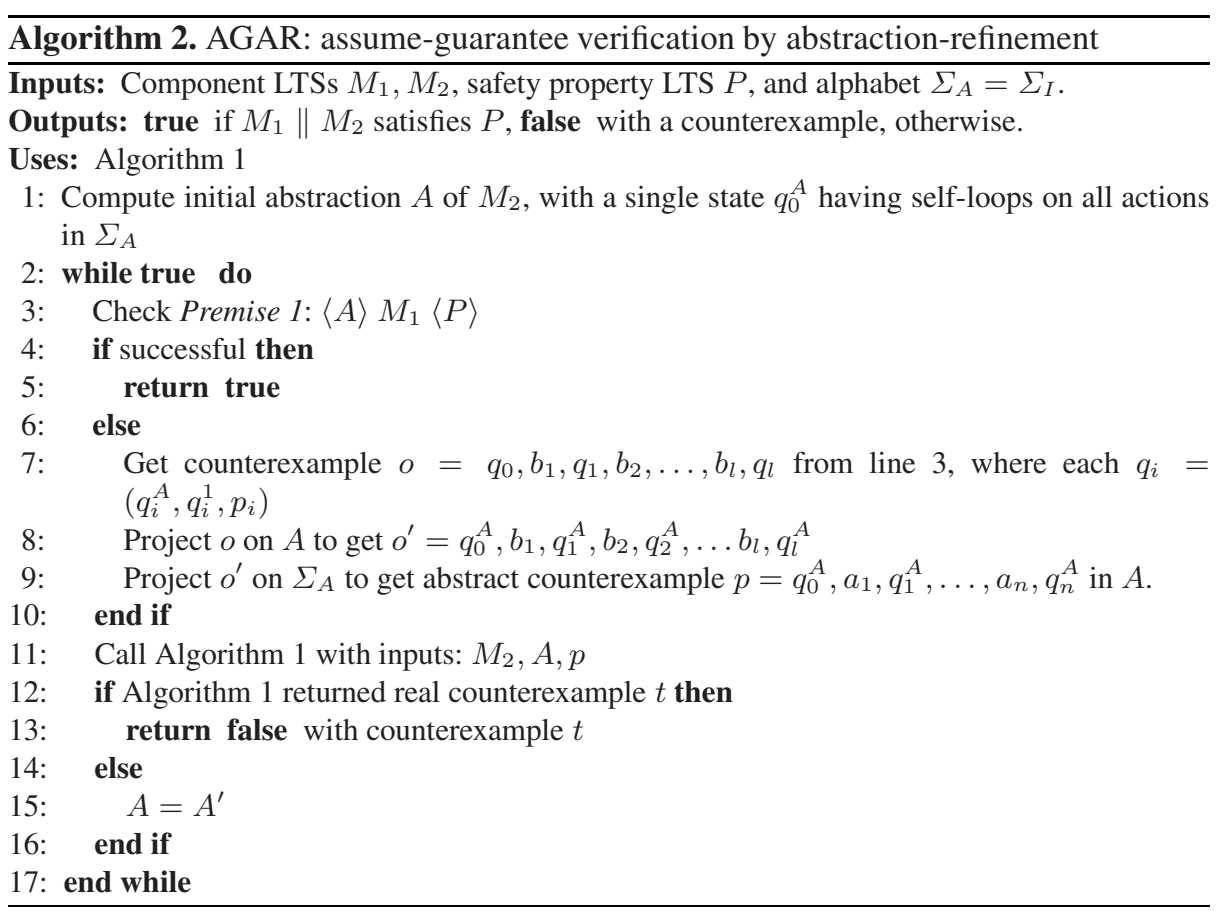

sequence, since they do not change the state of $A$; we eliminate from $o^{\prime}$ those actions and the duplicate abstract states that they connect, and finally obtain $p$ that we pass to Algorithm 1,

Theorem 1. Our algorithm (AGAR) computes a sequence of increasingly refined abstractions of $M_{2}$ until both premises of Rule $\square$ are satisfied, and we conclude that the property is satisfied by $M_{1} \| M_{2}$, or a real counterexample is found that shows the violation of the property on $M_{1} \| M_{2}$.

Proof. Correctness The algorithm terminates when Premise 1 is satisfied by the current abstraction or when a real counterexample is returned by Algorithm 1 In the former case, since the abstraction satisfies Premise 2 by construction (Proposition 1), Rule 1 ensures that $M_{1} \| M_{2}$ indeed satisfies $P$, so AGAR correctly returns answer "true". In the latter case, the counterexample returned by Algorithm 1 is a common trace of $M_{1}$ and of $M_{2}$ that leads to error in $P_{e r r}$. This shows that property $P$ is violated on $M_{1} \| M_{2}$ and in this case again AGAR correctly returns answer "false".

Termination. AGAR continues to refine the abstraction until a real counterexample is reported or the property holds. Refining the abstraction always results in a finer partition of its states (Lemma 1), and is thus guaranteed to terminate since in the worst case it converges to $M_{2}$ which is finite-state.

If $M_{2}$ has $n$ states, AGAR makes at most $n$ refinement iterations, and in each iteration, counterexample analysis performs at most $m$ closure operations, each of cost $O\left(n^{3}\right)$, 


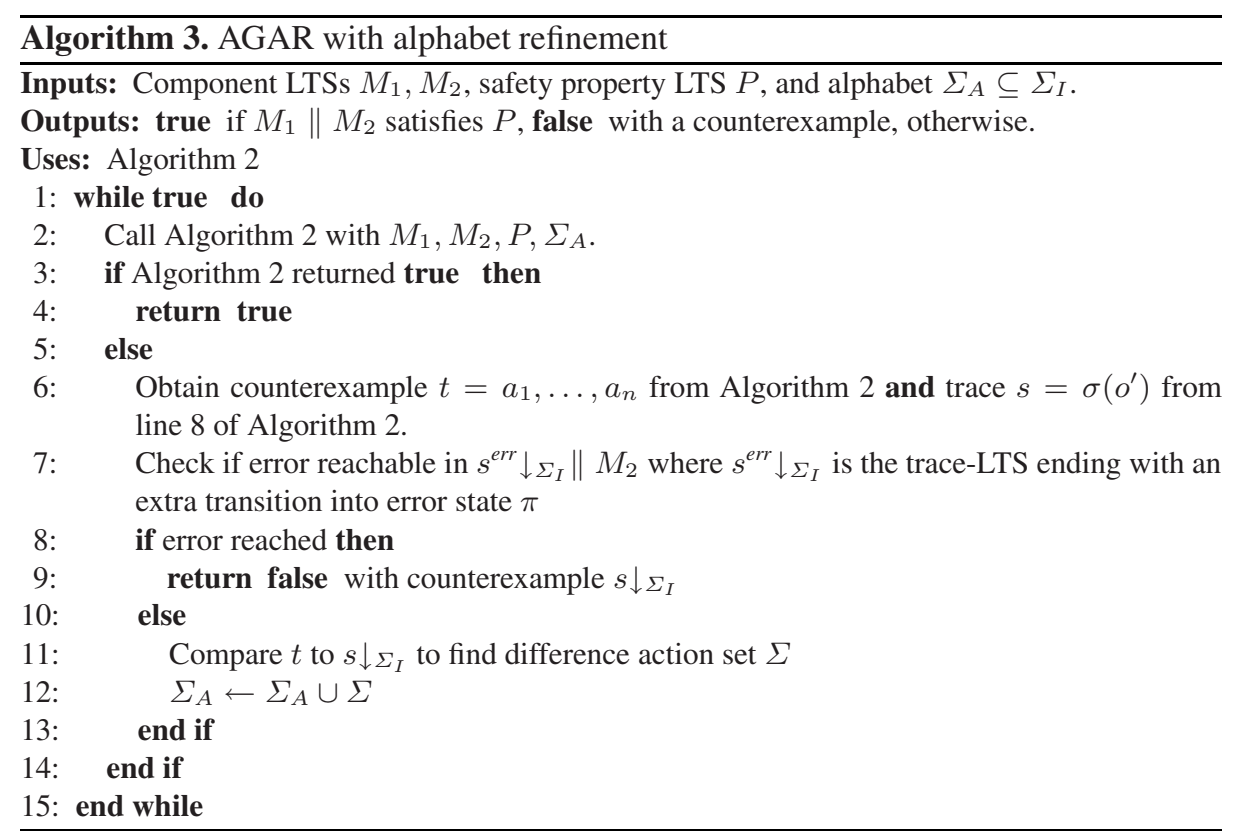

where $m$ is the length of the longest counterexample analyzed. This bound is not very tight as the closure steps are done on-the-fly to seldom exhibit worst-case behavior, and actually involve only parts of $M_{2}$ 's transition relation as needed.

\subsection{AGAR with Interface Alphabet Refinement}

In [11] we introduced an alphabet refinement technique to reduce the alphabet of the assumptions learned with $\mathrm{L}^{*}$. This technique improved significantly the performance of compositional verification. We show here how alphabet refinement can be similarly introduced in AGAR. Instead of the full interface alphabet $\Sigma_{I}$, we start AGAR from a small subset $\Sigma_{A} \subseteq \Sigma_{I}$. A good strategy is to start from those actions in $\Sigma_{I}$ that appear in the property to be verified, since the verification should depend on them. We then run Algorithm 2 with this small $\Sigma_{A}$. Alphabet refinement introduces an extra layer of approximation, due to the smaller alphabet being used.

The pseudocode is in Algorithm 3 . This algorithm adds an outer loop to AGAR (lines 1-15). At each iteration, it invokes AGAR (line 2) for the current alphabet $\Sigma_{A}$. If AGAR returns "true", it means that alphabet $\Sigma_{A}$ is enough for proving the property (and "true" is returned to the user). Otherwise, the returned counterexample needs to be further analyzed (lines 5-13) to see if it corresponds to a real error (which is returned to the user in line 9) or it is spurious due to the approximation introduced by the smaller interface alphabet, in which case it is used to refine this alphabet (lines 11-12).

Additional counterexample analysis. As explained in [11], when $\Sigma_{A} \subset \Sigma_{I}$, the counterexamples obtained by applying Rule 1 may be spurious, in which case $\Sigma_{A}$ needs to be extended. Intuitively, a counterexample is real if it is still a counterexample when 
Table 1. Comparison of AGAR and learning for 2 components, with and without alphabet refinement

\begin{tabular}{|c|c|c|c|c|c|c|c|c|c|c|c|c|c|c|c|}
\hline \multirow{3}{*}{ Case } & & \multicolumn{6}{|c|}{ No alpha. ref. } & \multicolumn{6}{|c|}{ With alpha. ref. } & \multirow{2}{*}{\multicolumn{2}{|c|}{ Sizes }} \\
\hline & & \multicolumn{3}{|c|}{ AGAR } & \multicolumn{3}{|c|}{ Learning } & \multicolumn{3}{|c|}{ AGAR } & \multicolumn{3}{|c|}{ Learning } & & \\
\hline & & $|A|$ & Mem. & Time & $A$ & Mem. & \begin{tabular}{|l|} 
Time \\
\end{tabular} & $A$ & Mem. & Time & $A$ & Mem. & Time & $P_{e r r} \|$ & $M_{2}$ \\
\hline Gas Station & 3 & $\begin{array}{l}16 \\
19 \\
22 \\
\end{array}$ & \begin{tabular}{|c|}
4.11 \\
37.43 \\
359.53
\end{tabular} & \begin{tabular}{|c|}
3.33 \\
23.12 \\
278.63 \\
\end{tabular} & $\begin{array}{l}177 \\
195 \\
45\end{array}$ & \begin{tabular}{|c|}
42.83 \\
100.17 \\
206.61
\end{tabular} & $\begin{array}{l}- \\
- \\
-\end{array}$ & \begin{tabular}{l|}
5 \\
5 \\
5
\end{tabular} & \begin{tabular}{|c|}
$\mathbf{2 . 9 9}$ \\
$\mathbf{2 2 . 7 9}$ \\
216.07
\end{tabular} & \begin{tabular}{c|}
2.09 \\
12.80 \\
83.34
\end{tabular} & $\begin{array}{l}8 \\
8 \\
8\end{array}$ & \begin{tabular}{|c|}
3.28 \\
25.21 \\
$\mathbf{2 0 7 . 2 9}$ \\
\end{tabular} & \begin{tabular}{|c|}
3.40 \\
19.46 \\
188.98 \\
\end{tabular} & $\begin{array}{c}1960 \\
16464 \\
134456\end{array}$ & \begin{tabular}{|c|}
643 \\
1623 \\
3447
\end{tabular} \\
\hline $\begin{array}{l}\text { Chiron, } \\
\text { Property } 2\end{array}$ & 2 & \begin{tabular}{|c|}
10 \\
36 \\
160 \\
4 \\
\end{tabular} & \begin{tabular}{|c|}
$\mathbf{1 . 3 0}$ \\
$\mathbf{2 . 5 9}$ \\
$\mathbf{8 . 7 1}$ \\
55.14 \\
\end{tabular} & \begin{tabular}{|c|}
$\mathbf{0 . 9 2}$ \\
$\mathbf{5 . 9 4}$ \\
152.34 \\
- \\
\end{tabular} & $\begin{array}{c}9 \\
21 \\
39 \\
111 \\
\end{array}$ & \begin{tabular}{|c|}
$\mathbf{1 . 3 0}$ \\
5.59 \\
27.1 \\
$\mathbf{5 6 9 . 2 3}$ \\
\end{tabular} & \begin{tabular}{|c|}
1.69 \\
7.08 \\
$\mathbf{3 2 . 0 5}$ \\
$\mathbf{6 7 6 . 0 2}$ \\
\end{tabular} & \begin{tabular}{|c|}
10 \\
36 \\
160 \\
3 \\
\end{tabular} & \begin{tabular}{|c|}
1.30 \\
$\mathbf{2 . 4 4}$ \\
$\mathbf{8 . 2 2}$ \\
58.71 \\
\end{tabular} & \begin{tabular}{|c}
$\mathbf{1 . 5 6}$ \\
$\mathbf{1 0 . 2 3}$ \\
252.06 \\
- \\
\end{tabular} & \begin{tabular}{|c|}
$\mathbf{8}$ \\
$\mathbf{2 0}$ \\
$\mathbf{3 8}$ \\
110 \\
\end{tabular} & \begin{tabular}{|c|}
$\mathbf{1 . 2 2}$ \\
6.00 \\
41.50 \\
- \\
\end{tabular} & \begin{tabular}{|c|}
5.17 \\
30.75 \\
$\mathbf{1 8 0 . 8 2}$ \\
386.6 \\
\end{tabular} & $\begin{array}{c}237 \\
449 \\
804 \\
2030 \\
\end{array}$ & $\begin{array}{c}102 \\
1122 \\
5559 \\
129228 \\
\end{array}$ \\
\hline $\begin{array}{l}\text { Chiron, } \\
\text { Property } 3\end{array}$ & $\overline{2}$ & $\begin{array}{c}4 \\
8 \\
16 \\
4\end{array}$ & \begin{tabular}{|c|}
1.07 \\
1.84 \\
4.01 \\
52.53
\end{tabular} & $\begin{array}{c}0.50 \\
1.60 \\
18.75 \\
-\end{array}$ & \begin{tabular}{|c|}
9 \\
$25 \mathrm{n}$ jmj \\
45 \\
$\mathbf{1 2 2}$ \\
\end{tabular} & \begin{tabular}{|c|}
1.14 \\
4.45 \\
25.49 \\
$\mathbf{1 3 4 . 2 1}$
\end{tabular} & \begin{tabular}{|c|}
1.57 \\
7.72 \\
36.33 \\
$\mathbf{2 7 1 . 3 0}$
\end{tabular} & $\begin{array}{c}4 \\
8 \\
16 \\
1\end{array}$ & \begin{tabular}{c|}
1.23 \\
$\mathbf{2 . 0 0}$ \\
$\mathbf{5 . 0 8}$ \\
81.89
\end{tabular} & \begin{tabular}{|c}
$\mathbf{0 . 6 2}$ \\
3.65 \\
107.50 \\
-
\end{tabular} & $\begin{array}{l}\mathbf{3} \\
3 \\
3 \\
3\end{array}$ & \begin{tabular}{|c|}
1.06 \\
2.28 \\
7.30 \\
$\mathbf{1 6 3 . 4 5}$
\end{tabular} & $\begin{array}{c}0.91 \\
\mathbf{1 . 1 2} \\
1.95 \\
\mathbf{1 9 . 4 3}\end{array}$ & $\begin{array}{c}258 \\
482 \\
846 \\
2084\end{array}$ & \begin{tabular}{|c|}
102 \\
1122 \\
5559 \\
129228
\end{tabular} \\
\hline$\overline{\text { MER }}$ & $\begin{array}{l}3 \\
4 \\
\end{array}$ & \begin{tabular}{|l|}
34 \\
67 \\
58 \\
\end{tabular} & \begin{tabular}{|c|}
$\mathbf{1 . 4 2}$ \\
$\mathbf{8 . 1 0}$ \\
341.49 \\
\end{tabular} & \begin{tabular}{|c|}
11.38 \\
247.73 \\
- \\
\end{tabular} & $\begin{array}{c}40 \\
335 \\
38 \\
\end{array}$ & \begin{tabular}{|c|}
6.75 \\
133.34 \\
377.21 \\
\end{tabular} & $\begin{array}{c}9.89 \\
- \\
- \\
\end{array}$ & \begin{tabular}{|l|}
5 \\
9 \\
9 \\
\end{tabular} & \begin{tabular}{|c|}
$\mathbf{1 . 4 2}$ \\
11.09 \\
532.49 \\
\end{tabular} & $\begin{array}{c}5.02 \\
180.13 \\
- \\
\end{array}$ & \begin{tabular}{c|}
6 \\
8 \\
10 \\
\end{tabular} & \begin{tabular}{|c|}
1.89 \\
$\mathbf{8 . 7 8}$ \\
$\mathbf{4 8 9 . 5 1}$ \\
\end{tabular} & \begin{tabular}{c||}
1.28 \\
12.56 \\
1220.62
\end{tabular} & $\begin{array}{c}143 \\
6683 \\
307623 \\
\end{array}$ & \begin{tabular}{|c|}
1270 \\
7138 \\
22886 \\
\end{tabular} \\
\hline Rover Exec. & 12 & $\overline{10}$ & $\overline{4.07}$ & $\overline{1.80}$ & 11 & 2.70 & 2.35 & $\overline{3}$ & 2.62 & 2.07 & $\overline{4}$ & $\overline{2.46}$ & 3.30 & $\overline{544}$ & $\overline{41}$ \\
\hline
\end{tabular}

considered with $\Sigma_{I}$. For counterexample analysis, we modify Algorithm 2 to also output the trace $s=\sigma\left(o^{\prime}\right)$ of actions along the intermediate path $o^{\prime}$ obtained at its line 8 . Since $p$ is a path obtained from $o^{\prime}$ by eliminating transitions labeled with actions from $\Sigma_{I} \backslash \Sigma_{A}$ (See Section 4.2) and $t=\sigma(p)$, it follows that $s$ is an "extension" of $t$ to $\Sigma_{I}$.

We check whether $s \downarrow_{\Sigma_{I}}$ is a trace of $M_{2}$ by making it into a trace LTS ending with the error state $\pi$, and whose alphabet is $\Sigma_{I}$ (line 7). Since $M_{2}$ does not contain $\pi$, the only way to reach error is if $s \downarrow_{\Sigma_{I}}$ is a trace of $M_{2}$; if we reach error, the counterexample $t$ is real. If $s \downarrow_{\Sigma_{I}}$ is not a trace of $M_{2}$, since $t$ is, we need to refine the current alphabet $\Sigma_{A}$. At this point we have two traces, $s \downarrow_{\Sigma_{I}}$ and $t$ that agree with respect to $\Sigma_{A}$ and only differ on the actions from $\Sigma_{I} \backslash \Sigma_{A}$; since one trace is in $M_{2}$ and the other is not, we are guaranteed to find in their symmetric difference at least an action that we can add to $\Sigma_{A}$ to eliminate the spurious counterexample $t$. We include the new action(s) and then repeat AGAR with the new alphabet. Termination follows from the fact that the interface alphabet is finite.

\section{Evaluation}

We implemented AGAR with alphabet refinement for Rule 1 in the LTSA tool. We compared AGAR with learning based assume guarantee reasoning, using a similar experimental setup as in [11]. The case studies are: Gas Station (with 3 . . 5 customers), Chiron - a model of a GUI (with $2 \ldots 5$ event handlers), and two NASA models: $M E R$ resource arbiter (with $2 \ldots 4$ threads competing for a common resource) and Rover, with an executive and an event monitoring component. We first used the same twoway decompositions of these models as described in [11]. For Gas Station and Chiron, these decompositions were demonstrated to be the best for the performance of learning (without alphabet refinement) among all possible two-way decompositions [9]. 
Table 2. Comparison of AGAR and learning for balanced decompositions

\begin{tabular}{|c|c|c|c|c|c|c|c|c|c|c|c|c|c|c|c|}
\hline \multirow{3}{*}{ Case } & \multirow{3}{*}{$k$} & \multicolumn{6}{|c|}{ No alpha. ref. } & \multicolumn{6}{|c|}{ With alpha. ref. } & \multirow{2}{*}{\multicolumn{2}{|c|}{$\begin{array}{r}1 \\
\text { Sizes }\end{array}$}} \\
\hline & & \multicolumn{3}{|c|}{ AGAR } & \multicolumn{3}{|c|}{ Learning } & \multicolumn{3}{|c|}{ AGAR } & \multicolumn{3}{|c|}{ Learning } & & \\
\hline & & $A$ & Mem. & Time & $A \mid$ & \begin{tabular}{|l|} 
Mem. \\
\end{tabular} & Time & $|A|$ & Mem. & Time & $A \mid$ & Mem. & Time & $M_{1}$ & $\mid M_{2}$ \\
\hline Gas Station & \begin{tabular}{|l|}
3 \\
4 \\
5 \\
\end{tabular} & \begin{tabular}{|c}
10 \\
269 \\
7 \\
\end{tabular} & \begin{tabular}{|c|}
$\mathbf{3 . 3 5}$ \\
174.03 \\
$\mathbf{4 7 . 9 1}$ \\
\end{tabular} & \begin{tabular}{|c|}
3.36 \\
- \\
184.64 \\
\end{tabular} & \begin{tabular}{|l|}
294 \\
433 \\
113 \\
\end{tabular} & \begin{tabular}{|c|}
367.13 \\
188.94 \\
82.59 \\
\end{tabular} & $\begin{array}{l}- \\
- \\
-\end{array}$ & $\begin{array}{c}5 \\
10 \\
2 \\
\end{array}$ & \begin{tabular}{|c|}
$\mathbf{2 . 1 6}$ \\
15.57 \\
47.48 \\
\end{tabular} & \begin{tabular}{|c|}
$\mathbf{3 . 0 6}$ \\
191.96 \\
- \\
\end{tabular} & \begin{tabular}{|c|}
59 \\
$\mathbf{5}$ \\
$\mathbf{1 5}$ \\
\end{tabular} & \begin{tabular}{|c|}
11.14 \\
$\mathbf{9 . 2 5}$ \\
$\mathbf{5 2 . 4 1}$ \\
\end{tabular} & \begin{tabular}{|c|}
81.19 \\
$\mathbf{4 . 7 3}$ \\
$\mathbf{7 1 . 2 9}$ \\
\end{tabular} & $\begin{array}{c}1692 \\
4608 \\
31411 \\
\end{array}$ & \begin{tabular}{|c|}
1942 \\
6324 \\
32768 \\
\end{tabular} \\
\hline $\begin{array}{l}\text { Chiron, } \\
\text { Property } 2\end{array}$ & $\begin{array}{l}2 \\
3 \\
4 \\
5\end{array}$ & \begin{tabular}{|c}
$\mathbf{4 1}$ \\
$\mathbf{2 6 1}$ \\
$\mathbf{5 4}$ \\
402 \\
\end{tabular} & \begin{tabular}{|c|}
$\mathbf{2 . 4 5}$ \\
$\mathbf{8 1 . 2 4}$ \\
$\mathbf{7 . 1 1}$ \\
73.74 \\
\end{tabular} & $\begin{array}{c}5.46 \\
710.1 \\
37.91 \\
- \\
\end{array}$ & \begin{tabular}{|l|}
140 \\
391 \\
354 \\
112
\end{tabular} & \begin{tabular}{|c|}
118.59 \\
134.57 \\
383.93 \\
90.22 \\
\end{tabular} & \begin{tabular}{|c|}
395.56 \\
- \\
- \\
- \\
\end{tabular} & $\begin{array}{c}9 \\
79 \\
45 \\
33 \\
\end{array}$ & \begin{tabular}{|c|}
1.91 \\
39.94 \\
9.55 \\
19.66 \\
\end{tabular} & \begin{tabular}{|c|}
3.89 \\
663.53 \\
121.66 \\
157.35 \\
\end{tabular} & \begin{tabular}{|c|}
17 \\
217 \\
586 \\
46
\end{tabular} & $\begin{array}{c}2.73 \\
36.12 \\
213.78 \\
30.05\end{array}$ & $\begin{array}{c}13.09 \\
- \\
- \\
686.37\end{array}$ & $\begin{array}{c}906 \\
6104 \\
1308 \\
11157\end{array}$ & \begin{tabular}{|c|}
924 \\
6026 \\
1513 \\
11748 \\
\end{tabular} \\
\hline $\begin{array}{l}\text { Chiron, } \\
\text { Property } 3\end{array}$ & \begin{tabular}{|l}
2 \\
3 \\
4 \\
5 \\
\end{tabular} & \begin{tabular}{|c}
2 \\
88 \\
2 \\
79 \\
\end{tabular} & \begin{tabular}{|c|}
0.98 \\
15.45 \\
5.60 \\
44.16 \\
\end{tabular} & \begin{tabular}{|c|}
$\mathbf{0 . 3 7}$ \\
$\mathbf{1 0 2 . 9 3}$ \\
$\mathbf{2 . 6 5}$ \\
405.03 \\
\end{tabular} & \begin{tabular}{|c|}
40 \\
184 \\
408 \\
179 \\
\end{tabular} & \begin{tabular}{|c|}
5.21 \\
284.83 \\
222.54 \\
104.25 \\
\end{tabular} & $\begin{array}{c}8.30 \\
- \\
- \\
-\end{array}$ & $\begin{array}{c}\mathbf{2} \\
46 \\
\mathbf{2} \\
42 \\
\end{array}$ & \begin{tabular}{|c|}
$\mathbf{1 . 0 2}$ \\
41.40 \\
$\mathbf{6 . 1 4}$ \\
42.04 \\
\end{tabular} & \begin{tabular}{|c|}
$\mathbf{0 . 4 9}$ \\
115.77 \\
11.90 \\
430.47 \\
\end{tabular} & $\begin{array}{c}3 \\
\mathbf{3} \\
20 \\
\mathbf{3} \\
\end{array}$ & $\begin{array}{c}1.04 \\
\mathbf{5 . 9 7} \\
9.33 \\
\mathbf{2 1 . 9 4} \\
\end{array}$ & $\begin{array}{l}0.91 \\
2.26 \\
7.44 \\
7.00\end{array}$ & $\begin{array}{c}168 \\
4240 \\
4156 \\
16431\end{array}$ & \begin{tabular}{|c|}
176 \\
4186 \\
4142 \\
16840 \\
\end{tabular} \\
\hline MER & 4 & $\overline{9}$ & 27.62 & $\overline{-}$ & 311 & 104.72 & - & $\overline{2}$ & 27.60 & - & $\overline{10}$ & $\overline{65.42}$ & $\overline{35.78}$ & 10045 & 66230 \\
\hline
\end{tabular}

All experiments were performed on a Dell PC with a $2.8 \mathrm{GHz}$ Intel Pentium $4 \mathrm{CPU}$ and a 1.0 GB RAM running Linux Fedora Core 4 and Sun's Java SDK version 1.5. We report the maximum assumption size (i.e., number of states) reached ("|A|"), the memory consumed ("Mem.") in MB, the time ("Time") in seconds, and the numbers of states on each side of the two-way decomposition: "| $M_{1} \| P_{\text {err }} \mid$ " and "| $M_{2} \mid "$. A "-" indicates that the limit of $1 \mathrm{G}$ of memory or 30 minutes has been exceeded. For those cases, the other quantities are shown as they were when the limit was reached. We also highlight in bold font the best results.

The results for the first set of experiments are shown in Table 1 Overall, AGAR shows similar or better results than learning in more than half of the cases. From the results, we noticed that the relative sizes of $M_{1} \| P_{\text {err }}$ and $M_{2}$ seem to influence the performance of the two algorithms; e.g., for Gas Station, where $M_{2}$ is consistently smaller, AGAR is consistently better, while for Chiron, as the size of $M_{2}$ becomes much larger, the performance of AGAR seems to degrade. Furthermore, we observed that the learning runs exercise more the first component, whereas AGAR exercises both. We therefore considered a second set of experiments were we tried to compare the relative performance of the two approaches for two-way system decompositions that are more balanced in terms of number of states.

We generated off-line all the possible two-way decompositions and chose those minimizing the difference in number of states between $M_{1} \| P_{\text {err }}$ and $M_{2}$. The rest of the setup remained the same. The results for these new decompositions are in Table 2 (for MER, in only one case we found a more balanced partition than previously). These results show that with these new decompositions AGAR is consistently better in terms of time (14/21 cases), memory (16/21 cases) and assumption size (16/21 cases) 2 . The results also indicate that the benefits of alphabet refinement are more pronounced for learning. The results are somewhat non-uniform as $k$ increases because for each larger value of $k$ we re-computed balanced decompositions independently of those for smaller values. This is why we even found smaller components for larger parameter, as for Chiron, Property $2, k=3$ vs. $k=4$.

\footnotetext{
${ }^{2}$ We did not count the cases when both algorithms ran out of limits.
} 


\section{Conclusions and Future Work}

We have introduced an assume-guarantee abstraction-refinement technique (AGAR) as an alternative to learning-based approaches. Our preliminary results clearly indicate that the alternative is feasible. We are currently extending AGAR with the following rule (for reasoning about $n$ components).

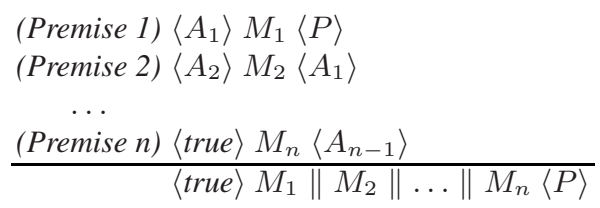

In previous work [11], learning with this rule overcame the intermediate state explosion related to two-way decompositions (i.e., when components are larger than the entire system). That helped us demonstrate better scalability of compositional vs. noncompositional verification which we believe to be the ultimate test of any compositional technique. We expect to similarly achieve better scalability for AGAR.

The implementation of AGAR for Rule 3 involves the creation of $n-1$ instances $A R_{i}$ of our abstraction-refinement code for computing each $A_{i}$ as an abstraction of $M_{i+1} \| A_{i+1}$, except for $A_{n-1}$ which abstracts $M_{n}$. Counterexamples obtained from (Premise 1) are used to refine the intermediate abstractions $A_{1}, \ldots, A_{n-1}$. When $A_{i}$ is refined, all the abstractions $A_{1}, \ldots, A_{i-1}$ are refined as well to eliminate the spurious trace. In the future, we also plan to explore extensions of AGAR to liveness properties.

Acknowledgements. We thank Moshe Vardi and Orna Grumberg for helpful suggestions and the CAV reviewers for their comments.

\section{References}

1. Alur, R., Madhusudan, P., Nam, W.: Symbolic Compositional Verification by Learning Assumptions. In: Etessami, K., Rajamani, S.K. (eds.) CAV 2005. LNCS, vol. 3576, pp. 548 562. Springer, Heidelberg (2005)

2. Angluin, D.: Learning regular sets from queries and counterexamples. Inf. and Comp. 75(2), 87-106 (1987)

3. Beyer, D., Henzinger, T.A., Singh, V.: Algorithms for Interface Synthesis. In: Damm, W., Hermanns, H. (eds.) CAV 2007. LNCS, vol. 4590, pp. 4-19. Springer, Heidelberg (2007)

4. Chaki, S., Clarke, E.M., Sinha, N., Thati, P.: Automated Assume-Guarantee Reasoning for Simulation Conformance. In: Etessami, K., Rajamani, S.K. (eds.) CAV 2005. LNCS, vol. 3576, pp. 534-547. Springer, Heidelberg (2005)

5. Chaki, S., Ouaknine, J., Yorav, K., Clarke, E.: Automated Compositional Abstraction Refinement for Concurrent C Programs: A Two-Level Approach. ENTCS 89(3) (2003)

6. Chaki, S., Strichman, O.: Optimized L*-Based Assume-Guarantee Reasoning. In: Grumberg, O., Huth, M. (eds.) TACAS 2007. LNCS, vol. 4424, pp. 276-291. Springer, Heidelberg (2007)

7. Clarke, E., Grumberg, O., Jha, S., Lu, Y., Veith, H.: Counterexample-Guided Abstraction Refinement. In: Emerson, E.A., Sistla, A.P. (eds.) CAV 2000. LNCS, vol. 1855, pp. 154169. Springer, Heidelberg (2000) 
8. Clarke, E.M., Grumberg, O., Peled, D.: Model Checking. MIT, Cambridge (2000)

9. Cobleigh, J.M., Avrunin, G.S., Clarke, L.A.: Breaking Up is Hard to Do: An Investigation of Decomposition for Assume-Guarantee Reasoning. In: Proc. of ISSTA 2006, pp. 97-108. ACM, New York (2006)

10. Cobleigh, J.M., Giannakopoulou, D., Pasareanu, C.S.: Learning Assumptions for Compositional Verification. In: ETAPS 2003 and TACAS 2003. LNCS, vol. 2619, pp. 331-346. Springer, Heidelberg (2003)

11. Gheorghiu, M., Giannakopoulou, D., Pasareanu, C.S.: Refining Interface Alphabets for Compositional Verification. In: Grumberg, O., Huth, M. (eds.) TACAS 2007. LNCS, vol. 4424, pp. 292-307. Springer, Heidelberg (2007)

12. Gupta, A., McMillan, K.L., Fu, Z.: Automated Assumption Generation for Compositional Verification. In: Damm, W., Hermanns, H. (eds.) CAV 2007. LNCS, vol. 4590, pp. 420-432. Springer, Heidelberg (2007)

13. Jones, C.B.: Specification and Design of (Parallel) Programs. In: Inf. Proc. 1983: Proc. of IFIP 9th World Congress, pp. 321-332. North Holland, Amsterdam (1983)

14. Magee, J., Kramer, J.: Concurrency: State Models \& Java Programs. John Wiley \& Sons, Chichester (1999)

15. Pnueli, A.: In Transition from Global to Modular Temporal Reasoning about Programs. Logic and Models of Conc. Sys. 13, 123-144 (1984)

16. Milner, R.: Communication and Concurrency. Prentice-Hall, New York (1989)

17. Sinha, N., Clarke, E.M.: SAT-Based Compositional Verification Using Lazy Learning. In: Damm, W., Hermanns, H. (eds.) CAV 2007. LNCS, vol. 4590, pp. 39-54. Springer, Heidelberg (2007) 